\title{
Pinyin Is an Effective Proxy for Early Screening for Mandarin-Speaking Children at Risk of Reading Disorders
}

\author{
Shaowei Ma ${ }^{1,2,3}$, Xiumei Zhang ${ }^{1}$, Hunter Hatfield ${ }^{2}$ and Wen-Hua Wej ${ }^{4 *}$ \\ ${ }^{1}$ School of Foreign Languages, Langfang Teachers University, Langfang, China, ${ }^{2}$ Department of English and Linguistics, \\ University of Otago, Dunedin, New Zealand, ${ }^{3}$ School of Teacher Education, College of Education, University of Canterbury, \\ Christchurch, New Zealand, ${ }^{4}$ Department of Women's and Children's Health, Dunedin School of Medicine, University \\ of Otago, Dunedin, New Zealand
}

\section{OPEN ACCESS}

Edited by:

Ann X. Huang,

Duquesne University, United States

Reviewed by:

Johannes Boltze,

University of Warwick,

United Kingdom

Franck Ramus,

Centre National de la Recherche

Scientifique (CNRS), France

*Correspondence:

Wen-Hua Wei

wenhua.wei@otago.ac.nz;

weiwedin05@gmail.com

Specialty section:

This article was submitted to

Educational Psychology,

a section of the journal

Frontiers in Psychology

Received: 07 October 2019

Accepted: 11 February 2020

Published: 26 February 2020

Citation:

Ma S, Zhang $X$, Hatfield $H$ and Wei W-H (2020) Pinyin Is an Effective

Proxy for Early Screening

for Mandarin-Speaking Children

at Risk of Reading Disorders.

Front. Psychol. 11:327.

doi: 10.3389/fpsyg.2020.00327
Reading disorders (RD) are common and complex neuropsychological conditions associated with decoding printed words and/or reading comprehension. Early identification of children at risk of $\mathrm{RD}$ is critical to allow timely interventions before mental suffering and reading impairment take place. Chinese is a unique medium for studying $\mathrm{RD}$ because of extra efforts required in reading acquisition of characters based on meaning rather than phonology. Pinyin, an alphabetic coding system mapping Mandarin sounds to characters, is important to develop oral language skills and a promising candidate for early screening for RD. In this pilot study, we used a cohort of 100 students (50 each in Grades 1 and 2) to derive novel profiles of applying Pinyin to identify early schoolers at risk of RD. Each student had comprehensive reading related measures in two consecutive years, including Pinyin reading and reading comprehension tested in the first and second year, respectively. We showed that Pinyin reading was mainly determined by phonological awareness, was well developed in Grade 1 and the top predictor of reading comprehension (explaining 30\% of variance, $p<1.0 \mathrm{e}-05$ ). Further, students who performed poorly in Pinyin reading [e.g. 1 standard deviation (SD) below the average, counting 14\% in Grade 1 and 10\% in Grade 2], tended to perform poorly in future reading comprehension tests, including all four individuals in Grade 1 (two out of three in Grade 2) who scored 1.5 SDs below the average. Pinyin is therefore an effective proxy for early screening for Mandarin-speaking children at risk of RD.

Keywords: Chinese reading, dyslexia, early screening, morphological awareness, phonological awareness, Pinyin, reading disorder

\section{INTRODUCTION}

Reading disorders (RD) are conditions occurring in learning to decode printed words (i.e. developmental dyslexia) and/or in learning to comprehend text (i.e. reading comprehension impairment) (Cutting et al., 2013; Hulme and Snowling, 2016; Snowling et al., 2019). Children with $\mathrm{RD}$ tend to have poor reading comprehension and thus low educational attainment and poor employment prospects (Hulme and Snowling, 2016; Snowling et al., 2019). Deciphering RD has 
been a long-standing challenge for over a century (Anderson and Meier-Hedde, 2001; Peterson and Pennington, 2015; Stein, 2018). This is largely owing to the high complexities in RD and the lack of coherent definitions across disciplines (Snowling, 2012; Snowling and Hulme, 2012; Stein, 2018). In essence, reading is a cognitive process of mapping letters/words to the sounds they represent in the brain. Hence RD can be considered as symptoms of neuropsychological disorders (Guerrini and Dobyns, 2014). With recent advances in many fields (e.g. neuroimaging), important progress has been made to understand causal mechanisms underlying the highly frequent RD (Pugh and Verhoeven, 2018; Facoetti et al., 2019). While mounting evidence indicate multiple causal links with $\mathrm{RD}$, further work is required to validate these hypothesized links (Gori et al., 2016; Facoetti et al., 2019).

Early identification of children at risk of reading failures is critical to allow timely interventions before the children suffer from potential downward spiral of underachievement, lowered self-esteem and poor motivation (Snowling, 2013). Indeed, during infancy and early childhood, human brains undergo fast development of spatial and temporal architecture and brain functions crucial for future performance ( $\mathrm{Li}$ et al., 2018). Early practices in the United Kingdom (Fawcett et al., 1998) and United States (Pennington and Lefly, 2001) indicate that pre-school screening tests of RD are feasible, leading to implementation of an "Early Years Foundation Profile Stage" in the United Kingdom (Snowling, 2013) and "Multi-Tier System of Supports" in the United States (Shepley and Grisham-Brown, 2019). These exercises together render a clear view that early oral language difficulties are strong predictors of later RD (Hulme and Snowling, 2016). Early screening for RD appears to be feasible in non-English language systems including Chinese (McBrideChang et al., 2008, 2011; Pugh and Verhoeven, 2018).

The Chinese language is believed to be a unique medium for studying RD (Tan et al., 2005; McBride et al., 2018). First, Chinese is a morpheme-based logographic system where each character is based on meaning rather than phonology and thus requires not only phonological but also morphological awareness (MA) for reading acquisition (Tan et al., 2005; Pan et al., 2016). Second, differences in the functional neurology (Tan et al., 2001; Zhao et al., 2017) and the genetic associations with reading were observed in Chinese populations (DeMille et al., 2018; Liu et al., 2019). Third, the RD trajectories appear different in Chinese populations (McBride et al., 2018). Nevertheless, cautions are recommended when interpreting results of $\mathrm{RD}$ studies from different Chinese communities where teaching systems, social settings for learning to read and $\mathrm{RD}$ diagnostic criteria are substantially different (McBride et al., 2018). For example, the estimated prevalence of dyslexia was $9.7 \%$ in Hong Kong (Chan et al., 2007) but $\sim 4 \%$ in mainland China (Liu et al., 2016; Zhao et al., 2016).

One typical difference is the introduction of Pinyin (meaning "spell sound"), a phonological coding system using Roman alphabet letters and four lexical tones to indicate the pronunciation of logographic characters, in mainland China but not Hong Kong (Wang et al., 2014; McBride et al., 2018). Pinyin is known to have multifold advantages in promoting Chinese reading: (a) using simple alphabetic transcripts to represent the sounds of Chinese characters; (b) bridging the spoken form with the written forms for each Chinese character and acting as a self-learning tool benefiting both new and experienced readers; (c) facilitating recognition of new characters through sublexical phonology (e.g. tone and syllable awareness); and (d) promoting memorizing and retrieving logographic characters via stable phonological cues (Lin et al., 2010; Wang et al., 2014; Ding et al., 2018). Given these advantages, Pinyin can be taught informally to kindergarteners as young as 3 years old and appears to be a good predictor of future Chinese reading performance (Lin et al., 2010; McBride-Chang et al., 2012; Yin and McBride, 2018). These factors together render Pinyin a potential valuable approach for early screening for children at risk of RD.

However, Pinyin is formally taught only in the first year of primary schools in mainland China (Wang et al., 2014; McBride et al., 2018). Several issues need to be addressed before any Pinyin screening applications become possible in Mandarin-speaking populations. One key issue is the lack of informative profiles illustrating how Pinyin reading proficiency post formal teaching may interplay with Chinese reading skills in the initial school years and particularly predict future reading failures. Previous evidence showed that poor readers in higher grades (e.g. Grade 4) did suffer more from Pinyin reading difficulties than normal readers (Yin and Weekes, 2003; Ding et al., 2015). This pilot study is therefore conducted to fill the information gap by re-analyzing the data generated from a project studying early Chinese reading development (Ma, 2016). We report characteristics of Pinyin reading measured after the formal Pinyin training in a study cohort and derive profiles of using Pinyin reading to identify children at risk of $\mathrm{RD}$ in early grades.

\section{MATERIALS AND METHODS}

\section{Participants}

One hundred children (50 in Grade 1 and 50 in Grade 2) from a state-funded mainstream school in Langfang (near Beijing, China) participated in this study, each with data measured in two consecutive academic years. All participants are native speakers of Mandarin - the official and instruction language in mainland China, where children normally start primary school around 7 years old and receive the full Pinyin training before learning to read Chinese characters. Pinyin teaching normally takes the first 12 weeks to cover onsets, rhymes, and lexical tones and spelling rules. The Pinyin phonetic symbols are continuously presented alongside Chinese characters in textbooks until Grade 3, and are provided only when new characters are introduced. None of the participants had obvious behavioral or emotional problems according to their class teachers. All participants were tested for receptive vocabulary using the Chinese version of Peabody Picture Vocabulary Test-Revised and appeared to have normal verbal intelligence (Lu and Liu, 1998).

\section{Procedure and Measures}

All reading related measures included in the study were administered to the participants individually by a trained 
examiner in a quiet room during the second semester of each academic year.

We included eight measures for phonological awareness (PA), four for MA, and four for rapid automatized naming (RAN) and Pinyin reading, which were measured for each participant in their first year of the study entry (i.e. school year 1 for Grades 1 and year 2 for Grade 2). We also included reading comprehension as the second outcome measure that was measured for each participant 1 year later after the study entry. We briefly describe these measures below. The full details and the summary statistics of these measures are available in Supplementary Note 1 and Supplementary Table 1, respectively.

\section{Reading Outcome Measures Pinyin reading}

A novel measure where children were asked to read out 50 onesyllable and 25 two-syllable words all in Pinyin scripts and scored for each syllable pronounced correctly. The maximum score is 75 .

\section{Reading comprehension}

This measure was designed following the model by Elbeheri et al. (2011) where children were asked to answer 36 multiple-choice questions silently in $15 \mathrm{~min}$ and scored for each correct answer. The maximum score is 36 .

\section{Phonological Awareness Measures}

Commonly used tests of phonological identification (Bradley and Bryant, 1983), deletion (McBride-Chang and Ho, 2000) and production (Chung et al., 2008) were employed to assess abilities to manipulate sounds at the syllabic, onset-rime and phonemic levels. For each measure, two practice tests were given prior to 15 formal tests to ensure sufficient understanding of how to perform the task properly, and children were scored for each correct answer in the formal test where no feedback was provided. The maximum score is 15 .

\section{Initial sound identification/deletion}

Children were asked to orally identify the odd initial sound from a set of three Chinese syllables provided with a same tone, or to orally delete the initial sound from a syllable provided.

\section{Final sound identification/deletion}

Children were asked to orally identify the odd final sound from a set of three Chinese syllables provided with a same tone, or to orally delete the final sound from a syllable provided.

\section{Rhyme detection/production}

Children were asked to orally identify the odd rhyme from a set of three Chinese syllables provided with a same tone, or to orally produce a real syllable with the same rhyme as that shared in the two Chinese syllables provided.

\section{Tone detection}

Children were asked to orally identify the odd tone from a set of three Chinese syllables (differ in both onsets and rimes) provided.

\section{Syllable identification}

Children were asked to orally identify the odd syllable from a set of three two-syllable Chinese words provided.

\section{Morphological Awareness Measures}

Commonly used tests of homograph discrimination ( $\mathrm{Ku}$ and Anderson, 2003) and production (Shu et al., 2006), homophone discrimination and production (Wenling et al., 2002) were employed to assess understanding of meaning and structure of compound words. For each measure, two practice tests were given prior to 15 formal tests to ensure sufficient understanding of how to perform the task properly, and children were scored for each correct answer in the formal test where no feedback was provided. The maximum score is 15 .

\section{Homograph discrimination/production}

Children were asked to orally identify the odd item with a unique meaning in the common morpheme shared in three two-character Chinese words provided, or to produce a twocharacter Chinese word with a different meaning from that in a common character shared by a pair of two-character Chinese words provided.

\section{Homophone discrimination/production}

Children were asked to orally identify the odd item with a unique meaning in the common homophonic morpheme shared by three two-character Chinese words provided, or to produce a two-character Chinese word with a different meaning from that in the homophonic syllable shared by a pair of two-character Chinese words provided.

\section{Rapid Automatized Naming Measures}

Four existing measures (Elbeheri et al., 2011; Liao et al., 2015) were adopted to assess ability to rapidly name graphological or non-graphological objects including digit naming testing of five single-digit integer numbers, picture naming testing of six color pictures of common objects, character naming testing of five simple Chinese characters, and Pinyin letter naming testing of five Pinyin letters. Only objects that are familiar to children were chosen in the tests. For each measure, one practice test was given prior to the formal test to ensure sufficient understanding of how to perform the task properly, and then children were instructed to read each object as fast and accurately as possible in the formal test and the time taken in pronouncing the given objects was recorded.

\section{Statistical Analysis}

All statistical analyses were conducted in $\mathrm{R}$ ( $\mathrm{R}$ Core Team, 2018) using packages available from https://cran.r-project.org/. In addition to summary statistics, pairwise Pearson correlations between the reading related measures were calculated using the cor() function and visualized using the corrplot() function in the corrplot package. Scatter plots were generated using the ggscatter() function in the corrplot package. Two additional sets of analyses were conducted to quantify impact of the cognitive measures on Pinyin reading and reading comprehension and to generate profiles of mock screening tests based on Pinyin reading.

\section{Factor Analysis and Linear Regression}

Exploratory factor analyses of measures in each of PA, MA, and RAN categories were performed, respectively, using the psych package in steps: (1) using VSS() to explore how many 
factors to be reduced from the given pairwise correlations; (2) using the factanal() to perform factor analysis by setting the reduced number of factors and the score method as "Bartlett"; and (3) storing the resultant factor scores for further analyses. Linear regressions and subsequent analyses of variance were performed to assess relative impact of PA, MA, and RAN factors on Pinyin reading and reading comprehension using $\operatorname{lm}()$ and anova(), respectively. Variance inflation factor and tolerance of multicollinearity were assessed for each regression model using ols_vif_tol() in the olsrr package. The $\mathrm{R}$ scripts used in the analyses and relevant details are available in Supplementary Note 2.

\section{Mock Screening Test Analysis}

Using a threshold of 1 SD below the average in Pinyin reading, mock screening tests were performed for students in Grades 1 and 2, respectively. Assuming those performed 1.5 SDs below the average in reading comprehension to be the "true" cases with $\mathrm{RD}$, the screening test results were analyzed to derive (1) a screening out rate, calculated as the number of screened out divided by the total number of samples, (2) screen out true positive rate, calculated as the number of cases screened out divided by the total number of cases, and (3) screen out false positive rate, calculated as the number of non-cases divided by the total number of individuals screened out.

\section{RESULTS}

All reading related measures included in the study were informative with clear variations (Supplementary Table 1). These measures were often highly correlated and their correlations varied with grades (Supplementary Figure 1). Pinyin reading was strongly correlated with almost every reading related measure in Grade 1, and the correlations remained strong in Grade 2 except for those with the MA measures, indicating a great potential of Pinyin for early screening for RD in school students. Most PA measures were strongly correlated with other measures relatively consistently across grades, whereas the correlations between the MA and the RAN measures were generally weak/moderate (Supplementary Figure 1).

Exploratory factor analyses were performed for each category of reading related measures to simplify the complex correlation structures, and resulted in 1-factor solutions for every category and standardized factor scores named as PAscore, MAscore, and RANscore, respectively (Supplementary Note 2). The distributions of the factor scores showed clearly that, compared with the counterparts in Grade 1, MAscore and RANscore were substantially improved in Grade 2 whereas PAscore remained relatively stable (Figure 1), suggesting PA measures were probably well developed in Grade 1.

Variance analyses showed that PAscore was indeed the top predictor of Pinyin reading, contributing $71.8 \%$ of the total variance $(p=1.4 \mathrm{e}-15)$ in Grade 1 and $47 \%(p=1.9 \mathrm{e}-09)$ in Grade 2 (Table 1). RANscore was also a significant predictor of Pinyin reading but only in Grade 2, contributing $15.7 \%$ of the total variance $(p=7.9 \mathrm{e}-05)$. For reading comprehension,

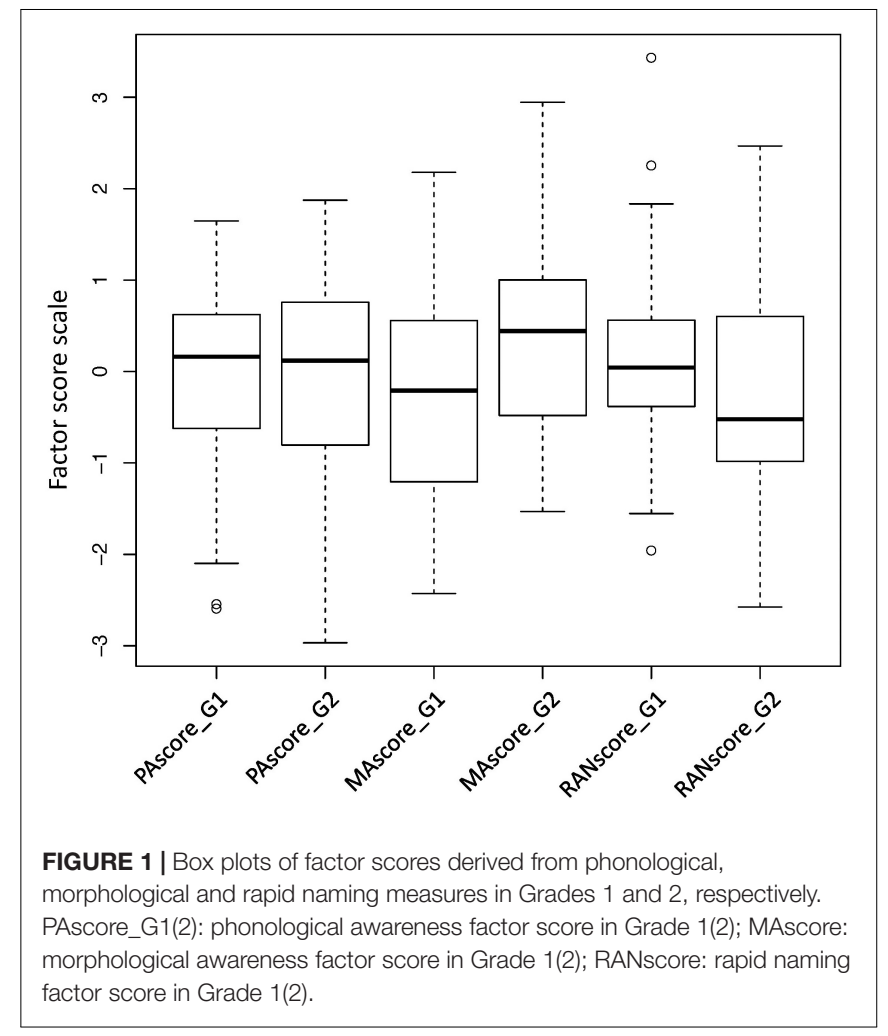

when fitting Pinyin reading as the first covariate followed by the factor scores, Pinyin reading was the top predictor explaining $33.9 \%$ of the total variance $(p=1.2 \mathrm{e}-06)$ in Grade 1 and $29.9 \%(p=7.8 \mathrm{e}-06)$ in Grade 2 (Table 1). Despite the collinearity with Pinyin reading, PAscore remained the second predictor of reading comprehension, contributing $10.4 \%$ of the total variance $(p=0.003)$ in Grade 1 and $7.5 \%$ $(p=0.015)$ in Grade 2, whereas RANscore again contributed significantly only in Grade $2(9.6 \%, p=0.006)$. When fitting Pinyin reading as the last covariate after the factor scores, Pinyin reading became the least predictor explaining little additional variance of reading comprehension in either Grade as expected. These results jointly suggest that Pinyin reading statistically is a good proxy for the PA, MA and RAN measures (Supplementary Figure 2).

We then visually examined the distributions of Pinyin reading against reading comprehension (Figure 2). While the performances in the two outcome readings corresponded in general, the data points at the bottom left (poor performers in both) were much sparser than those at the top right (good performers in both) in each plot, indicating that most students underwent healthy development of Chinese reading and Pinyin reading could indeed pick up poor readers, particularly in Grade 1 where students were just in their second year of school when reading comprehension was measured and thus were still within the early stage of reading development as evidenced by wide variation in reading comprehension. In contrast, in Grade 2, data points clearly clustered in two groups by either reading outcome, but Pinyin reading became less indicative with outliers 
TABLE 1 | Variance explained by each attribute in reading outcome*.

\begin{tabular}{|c|c|c|c|c|c|c|c|c|}
\hline \multirow[b]{3}{*}{ Attribute } & \multicolumn{4}{|c|}{ Pinyin reading } & \multicolumn{4}{|c|}{ Reading comprehension } \\
\hline & \multicolumn{2}{|c|}{ Grade 1} & \multicolumn{2}{|c|}{ Grade 2} & \multicolumn{2}{|c|}{ Grade 1} & \multicolumn{2}{|c|}{ Grade 2} \\
\hline & Variance $\%$ & $p$ & Variance \% & $p$ & Variance $\%$ & $p$ & Variance $\%$ & $p$ \\
\hline Pinyin reading & $\mathrm{n} / \mathrm{a}$ & $\mathrm{n} / \mathrm{a}$ & $\mathrm{n} / \mathrm{a}$ & $\mathrm{n} / \mathrm{a}$ & $33.9 \%$ & $1.2 \mathrm{e}-06$ & $29.9 \%$ & $7.8 \mathrm{e}-06$ \\
\hline PAscore & $71.8 \%$ & $1.4 \mathrm{e}-15$ & $47.0 \%$ & $1.9 \mathrm{e}-09$ & $10.4 \%$ & 0.003 & $7.5 \%$ & 0.015 \\
\hline MAscore & $0.0 \%$ & 0.787 & $0.8 \%$ & 0.343 & $6.8 \%$ & 0.016 & $1.8 \%$ & 0.224 \\
\hline RANscore & $1.9 \%$ & 0.060 & $15.7 \%$ & $7.9 e-05$ & $0.4 \%$ & 0.569 & $9.6 \%$ & 0.006 \\
\hline
\end{tabular}

*Gender is not important in any models; age was significant $(p=0.009)$ in the Grade 1 model of Pinyin reading only, accounting for $3.7 \%$ of total variance.

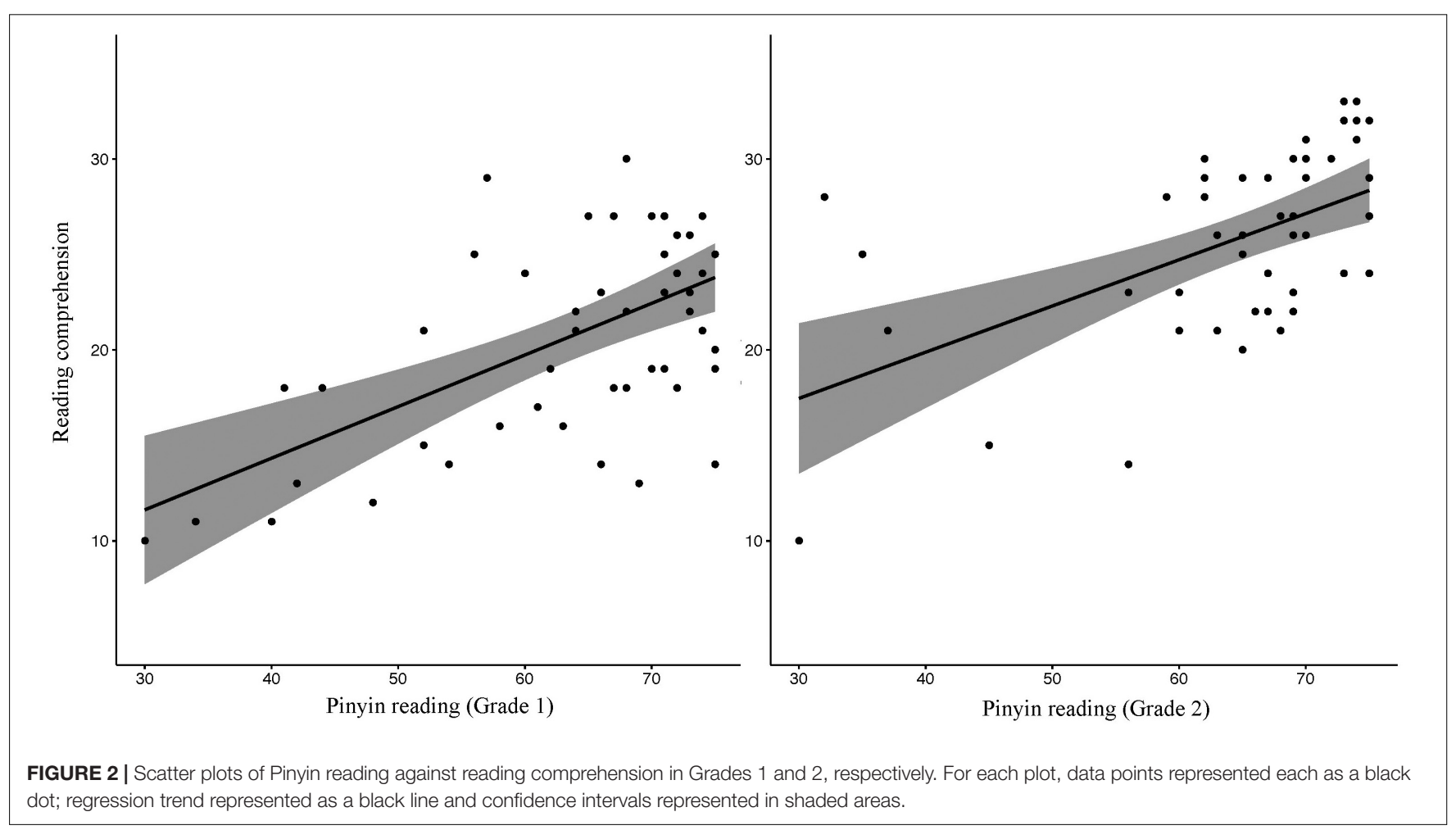

in both directions (Figure 2) possibly because of other reading developments (e.g. RAN skills) in these older students.

Assuming students with 1.5 SDs below the average of reading comprehension as $\mathrm{RD}$ cases, we further performed mock screening tests using a threshold of $1 \mathrm{SD}$ below the average of Pinyin reading (Table 2). The mock test in Grade 1 identified 7 (14\%) students as being at risk of RD including all four predefined RD cases, and thus had $100 \%$ screen out true positive rate in early detection of RD. Of the remaining three classified as false positives, S32 could well be a RD case because the student had a reading comprehension of 13 slightly above the threshold of 12.6 but had the poorest RANscore and the second poorest PAscore (Supplementary Table 2). The mock test in Grade 2 identified 5 (10\%) students including only 2 of the 3 predefined RD cases, where the missed RD case S57 was the oldest student who had the second poorest RANscore and all three false positives (i.e. S66, S71, and S81) had a poor RANscore coincidently (Supplementary Table 3).

\section{DISCUSSION}

In this pilot study, we ascertained the feasibility of using Pinyin reading to screen cohorts of early schoolers for individuals at risk of $\mathrm{RD}$. Within the data collected, all predefined poor readers were screened out by simply applying a threshold of 1 SD below the average of Pinyin reading in Grade 1. The screening tests identified less than $15 \%$ of students as being at risk for RD, which is a strong first step in identifying those in need for early intervention and full assessment. Furthermore, implementation of the Pinyin screening is convenient and cost effective given Pinyin training is compulsory for every new primary school student in mainland China. Ideally, such implementations could happen immediately after the Pinyin teaching in order to maximize the window of effective interventions under the current teaching system.

Applying Pinyin early screening in Grade 1 is also endorsed by the facts that Pinyin reading was mostly determined by 
TABLE 2 | Pinyin mock screening test results.

\begin{tabular}{lcc}
\hline Item & Grade 1 & Grade 2 \\
\hline Total samples with data & 50 & 49 \\
Threshold of Pinyin reading & 52.0 & 53.3 \\
Threshold of reading comprehension & 12.6 & 18.45 \\
Number of RD cases & 4 & 3 \\
Total screened out & 7 & 5 \\
RD cases screened out & 4 & 2 \\
Screening out rate & $14 \%$ & $10 \%$ \\
Screen out true positive rate & $100 \%$ & $67 \%$ \\
Screen out false positive rate & $43 \%$ & $60 \%$ \\
\hline
\end{tabular}

PA and both were well developed in Grade 1 with only small changes of means in Grade 2 (Figure 1 and Supplementary Table 1). In contrast, morphological awareness had a late onset of development to learn Chinese characters and meanings (McBride et al., 2018), and thus had limited values in predicting either Pinyin reading or reading comprehension at this early school stage (Table 1). However, the influence of MAscore might have been offset partially by PAscore in the regression model of reading comprehension because of their correlations (Table 1 and Supplementary Figure 1) and the unique mediation relationship between syllable awareness (phonological) and morphological awareness in Chinese (Pan et al., 2016). Similarly, rapid naming skills had limited values in predicting reading outcome in Grade 1 but became important in Grade 2, as shown in previous studies (Lervag and Hulme, 2009; Liao et al., 2015).

Can Pinyin early screening be implemented at the preschool stage? The answer is probably yes given the reasons above and the successful examples of English early screening in the United Kingdom and United States, each also relying heavily on PA (Snowling, 2013; Shepley and Grisham-Brown, 2019). However, further investigations of Pinyin applications at the kindergarten stage are needed to generate comprehensive and coherent evidence for policy makers in mainland China, as showed in the development of the English examples (Snowling, 2013). One obvious discrepancy is that Pinyin reading of early schoolers (i.e. 7 or 8 years old) explained $\sim 30 \%$ of the reading comprehension variance (Table 1), whereas Pinyin measures (e.g. Pinyin invented spelling) of kindergarteners could explain less than $10 \%$ of the variance of future Chinese reading (McBrideChang et al., 2012; Yin and McBride, 2018). One main reason for the discrepancy could be that only simple Pinyin skills can be taught informally in kindergartens. Therefore, it is essential to develop standardized procedures for Pinyin teaching and assessment for kindergarteners.

Although only a small sample of an ordinary school in China was used in this pilot study, the observed rates of students at risk of $\mathrm{RD}$ (i.e. 8 and $6 \%$ in Grades 1 and 2, respectively, Table 2) however, are in line with the hypothesis that dyslexia could be less prevalent in Mandarin-speaking communities as reported previously (Dai et al., 2016; Liu et al., 2016; Zhao et al., 2016) than in Hong Kong (9.7\%) (Chan et al., 2007) where Pinyin is not used in Chinese teaching. In addition to different diagnostic criteria used, differences in education systems and special social settings for learning in Chinese populations could also explain the discrepancy in the reported prevalence of dyslexia. For example, Confucianism-based motivation leads to preschool education and private trainings (e.g. music, painting) commonly adopted in mainland China, which may virtually act as effective interventions and thus reduce the prevalence of dyslexia often measured at school age (Dai et al., 2016; Pan et al., 2017; McBride et al., 2018). Besides, Pinyin learning could be another hidden intervention since the key cognitive-linguistic skills for learning Chinese (e.g. phonological sensitivity) may be initially integrated in Pinyin training (Wang et al., 2014; McBride et al., 2018). Further investigations of differences in dyslexia prevalence across the Chinese communities and any additional roles of Pinyin (Ding et al., 2018; Chen et al., 2019) are therefore warranted.

Successful early screening for children at risk of $\mathrm{RD}$ could boost genetic studies that are limited mainly by small samples available and heterogeneity in phenotyping across ethnic communities (DeMille et al., 2018; Liu et al., 2019). With small at-risk groups, it is economically feasible to apply new but expensive technologies such as functional and structural magnetic resonance imaging (Skeide et al., 2015, 2016; Kraft et al., 2016) to improve diagnoses as well as characterization of intermediate features (e.g. working-memory and hearing) highly associated with RD (Mannel et al., 2015; Neef et al., 2017). These together will greatly increase the number of well-defined RD cases and consequently the power of genetic association studies, which in turn will enable genetic prediction of the RD risk (Muller et al., 2016). Furthermore, these could promote cross-population dissection of the genetic mechanisms underlying RD by metaanalyzing data derived from Chinese and European samples and eventually identify any Chinese-specific genetic variants (Rosenberg et al., 2010; Liu et al., 2019).

Nonetheless, cautions are recommended when interpreting the results of this pilot study that is limited by small samples and hypothesized statistical analyses without actual diagnosis of RD in any samples. Using the threshold of 1 SD (instead of 1.5 SD) below the average of Pinyin reading was to ensure all RD cases were found in early screening for Grade 1 . The estimates of screen out true positive rate and percentages of students at risk of RD based on such a small sample size can only be indicative at most. Large and well-designed longitudinal cohort studies are needed to generate accurate profiles for Pinyin screening tests at both the school and pre-school stage. Such studies will simultaneously benefit genetic epidemiology studies of dyslexia in China.

\section{CONCLUSION}

Pinyin is an effective proxy for early screening for Mandarinspeaking children at risk of $\mathrm{RD}$.

\section{DATA AVAILABILITY STATEMENT}

The datasets generated for this study are available on request to the corresponding author. 


\section{ETHICS STATEMENT}

Participation in this study was voluntary and with parental/guardian written informed consent. Ethics approval for this study was provided by the Ethics Committee of the University of Canterbury (reference number: 2012/56/ERHEC).

\section{AUTHOR CONTRIBUTIONS}

$\mathrm{SM}, \mathrm{HH}$, and $\mathrm{W}-\mathrm{HW}$ designed the study. SM and $\mathrm{W}-\mathrm{HW}$ analyzed the data and prepared the draft and final manuscript. SM collected the data and performed the study. XZ assisted the data collection and contributed to the manuscript writing. $\mathrm{HH}$ contributed to the data analysis and manuscript writing.

\section{FUNDING}

This study was facilitated by New Zealand-China NonCommunicable Diseases Collaboration Research Centre that is funded by the Ministry of Business, Innovation and Employment, New Zealand. SM acknowledges salary and research funding

\section{REFERENCES}

Anderson, P. L., and Meier-Hedde, R. (2001). Early case reports of dyslexia in the United States and Europe. J. Learn. Disabil. 34, 9-21. doi: 10.1177/ 002221940103400102

Bradley, L., and Bryant, P. E. (1983). Categorizing sounds and learning to read-a causal connection. Nature 301, 419-421. doi: 10.1038/301419a0

Chan, D. W., Ho, C. S. H., Tsang, S. M., Lee, S. H., and Chung, K. K. H. (2007). Prevalence, gender ratio and gender differences in reading-related cognitive abilities among Chinese children with dyslexia in Hong Kong. Educ. Stud. 33, 249-265. doi: 10.1080/03055690601068535

Chen, L., Perfetti, C. A., Fang, X., Chang, L.-Y., and Fraundorf, S. (2019). Reading Pinyin activates sublexcial character orthography for skilled Chinese readers. Lang. Cogn. Neurosci. 34, 736-746. doi: 10.1080/23273798.2019.157 8891

Chung, K. K. H., McBride-Chang, C., Wong, S. L., Cheung, H., Penney, T. B., and Ho, C. S. H. (2008). The role of visual and auditory temporal processing for Chinese children with developmental dyslexia. Ann. Dyslexia 58, 15-35. doi: 10.1007/s11881-008-0015-4

Cutting, L. E., Clements-Stephens, A., Pugh, K. R., Burns, S., Cao, A., Pekar, J. J., et al. (2013). Not all reading disabilities are dyslexia: distinct neurobiology of specific comprehension deficits. Brain Connect. 3, 199-211. doi: 10.1089/brain. 2012.0116

Dai, L., Zhang, C., and Liu, X. (2016). A special Chinese reading acceleration training paradigm: to enhance the reading fluency and comprehension of chinese children with reading disabilities. Front. Psychol. 7:1937. doi: 10.3389/ fpsyg.2016.01937

DeMille, M. M. C., Tang, K., Mehta, C. M., Geissler, C., Malins, J. G., Powers, N. R., et al. (2018). Worldwide distribution of the DCDC2 READ1 regulatory element and its relationship with phoneme variation across languages. Proc. Natl. Acad. Sci. U.S.A. 115, 4951-4956. doi: 10.1073/pnas.1710472115

Ding, Y., Liu, R. D., McBride, C., and Zhang, D. (2015). Pinyin invented spelling in mandarin chinese-speaking children with and without reading difficulties. J. Learn. Disabil. 48, 635-645. doi: 10.1177/0022219414522704

Ding, Y., Liu, R.-D., McBride, C. A., Fan, C.-H., Xu, L., and Wang, J. (2018). Pinyin and english invented spelling in chinese-speaking students who speak english as a second language. J. Psycholinguist. Res. 47, 1163-1187. doi: 10.1007/s10936018-9585-4 support from the Langfang Teachers University, and a travel grant from the University of Canterbury to support the data collection in China. W-HW was funded by the Cure Kids New Zealand and the University of Otago.

\section{ACKNOWLEDGMENTS}

The authors would like to thank all the school children, teachers, and examiners who participated in the study. The authors are grateful for the data available from for SM's Ph.D. project that was supervised by Profs. John Everatt and Brigid McNeill at the University of Canterbury, NZ. The authors greatly appreciate the valuable comments and suggestions from the two reviewers that substantially improve the readability of the manuscript.

\section{SUPPLEMENTARY MATERIAL}

The Supplementary Material for this article can be found online at: https://www.frontiersin.org/articles/10.3389/fpsyg. 2020.00327/full\#supplementary-material

Elbeheri, G., Everatt, J., Mahfoudhi, A., Abu Al-Diyar, M., and Taibah, N. (2011). Orthographic processing and reading comprehension among arabic speaking mainstream and LD children. Dyslexia 17, 123-142. doi: 10.1002/dys.430

Facoetti, A., Gori, S., Vicari, S., and Menghini, D. (2019). Introduction to the special issue: developmental dyslexia: from genes to remediation. Neuropsychologia 130, 1-2. doi: 10.1016/j.neuropsychologia.2019.06.003

Fawcett, A. J., Singleton, C. H., and Peer, L. (1998). Advances in early years screening for dyslexia in the United Kingdom. Ann. Dyslexia 48, 57-88. doi: 10.1007/s11881-998-0004-7

Gori, S., Seitz, A. R., Ronconi, L., Franceschini, S., and Facoetti, A. (2016). Multiple causal links between magnocellular-dorsal pathway deficit and developmental dyslexia. Cereb. Cortex 26, 4356-4369. doi: 10.1093/cercor/bhv206

Guerrini, R., and Dobyns, W. B. (2014). Malformations of cortical development: clinical features and genetic causes. Lancet Neurol. 13, 710-726. doi: 10.1016/ S1474-4422(14)70040-7

Hulme, C., and Snowling, M. J. (2016). Reading disorders and dyslexia. Curr. Opin. Pediatr. 28, 731-735. doi: 10.1097/mop.0000000000000411

Kraft, I., Schreiber, J., Cafiero, R., Metere, R., Schaadt, G., Brauer, J., et al. (2016). Predicting early signs of dyslexia at a preliterate age by combining behavioral assessment with structural MRI. Neuroimage 143, 378-386. doi: 10.1016/j. neuroimage.2016.09.004

Ku, Y.-M., and Anderson, R. C. (2003). Development of morphological awareness in Chinese and English. Read. Writ. 16, 399-422. doi: 10.1023/a:1024227231216

Lervag, A., and Hulme, C. (2009). Rapid automatized naming (RAN) taps a mechanism that places constraints on the development of early reading fluency. Psychol. Sci. 20, 1040-1048. doi: 10.1111/j.1467-9280.2009.02405.x

Li, M., Santpere, G., Imamura Kawasawa, Y., Evgrafov, O. V., Gulden, F. O., Pochareddy, S., et al. (2018). Integrative functional genomic analysis of human brain development and neuropsychiatric risks. Science 362:eaat7615. doi: 10. 1126/science.aat7615

Liao, C. H., Deng, C., Hamilton, J., Lee, C. S., Wei, W., and Georgiou, G. K. (2015). The role of rapid naming in reading development and dyslexia in Chinese. J. Exp. Child Psychol. 130, 106-122. doi: 10.1016/j.jecp.2014.10.002

Lin, D., McBride-Chang, C., Shu, H., Zhang, Y. P., Li, H., Zhang, J. A., et al. (2010). Small wins big: analytic pinyin skills promote chinese word reading. Psychol. Sci. 21, 1117-1122. doi: 10.1177/0956797610375447

Liu, L., Gu, H., Hou, F., Xie, X., Li, X., Zhu, B., et al. (2019). Dyslexia associated functional variants in Europeans are not associated with dyslexia in Chinese. 
Am. J. Med. Genet. B Neuropsychiatr. Genet. 180, 488-495. doi: 10.1002/ajmg.b. 32750

Liu, L., Wang, J., Shao, S., Luo, X., Kong, R., Zhang, X., et al. (2016). Descriptive epidemiology of prenatal and perinatal risk factors in a Chinese population with reading disorder. Sci. Rep. 6:36697. doi: 10.1038/srep36697

Lu, L., and Liu, H. H. (1998). Chinese Version of Peabody Picture Vocabulary Test-Revised. Taipei: Psychological Publishing.

Ma, S. (2016). Comparisons of the Influence of Phonological and Morphological Processing on Chinese Reading Development: A Cross-Sectional and Longitudinal Study. Ph.D. thesis, University of Canterbury, Christchurch.

Mannel, C., Meyer, L., Wilcke, A., Boltze, J., Kirsten, H., and Friederici, A. D. (2015). Working-memory endophenotype and dyslexia-associated genetic variant predict dyslexia phenotype. Cortex 71, 291-305. doi: 10.1016/j.cortex. 2015.06.029

McBride, C., Wang, Y., and Cheang, L. M.-L. (2018). Dyslexia in Chinese. Curr. Dev. Disord. Rep. 5, 217-225. doi: 10.1007/s40474-018-0149-y

McBride-Chang, C., and Ho, C. S. H. (2000). Developmental issues in Chinese children's character acquisition. J. Educ. Psychol. 92, 50-55. doi: 10.1037//00220663.92.1.50

McBride-Chang, C., Lam, F., Lam, C., Chan, B., Fong, C. Y. C., Wong, T. T. Y., et al. (2011). Early predictors of dyslexia in Chinese children: familial history of dyslexia, language delay, and cognitive profiles. J. Child Psychol. Psychiatry 52, 204-211. doi: 10.1111/j.1469-7610.2010.02299.x

McBride-Chang, C., Lam, F., Lam, C., Doo, S., Wong, S. W. L., and Chow, Y. Y. Y. (2008). Word recognition and cognitive profiles of Chinese pre-school children at risk for dyslexia through language delay or familial history of dyslexia. J. Child Psychol. Psychiatry 49, 211-218. doi: 10.1111/j.1469-7610.2007.01837.x

McBride-Chang, C., Lin, D., Liu, P. D., Aram, D., Levin, I., Cho, J. R., et al. (2012). The ABC's of Chinese: maternal mediation of Pinyin for Chinese children's early literacy skills. Read. Writ. 25, 283-300. doi: 10.1007/s11145-010-9270-y

Muller, B., Wilcke, A., Boulesteix, A. L., Brauer, J., Passarge, E., Boltze, J., et al. (2016). Improved prediction of complex diseases by common genetic markers: state of the art and further perspectives. Hum. Genet. 135, 259-272. doi: 10. 1007/s00439-016-1636-z

Neef, N. E., Muller, B., Liebig, J., Schaadt, G., Grigutsch, M., Gunter, T. C., et al. (2017). Dyslexia risk gene relates to representation of sound in the auditory brainstem. Dev. Cogn. Neurosci. 24, 63-71. doi: 10.1016/j.den.2017.01.008

Pan, J., Kong, Y., Song, S., McBride, C., Liu, H., and Shu, H. (2017). Socioeconomic status, parent report of children's early language skills, and late literacy skills: a long term follow-up study among Chinese children. Read. Writ. 30, 401-416. doi: 10.1007/s11145-016-9682-4

Pan, J., Song, S., Su, M., McBride, C., Liu, H., Zhang, Y., et al. (2016). On the relationship between phonological awareness, morphological awareness and Chinese literacy skills: evidence from an 8-year longitudinal study. Dev. Sci. 19, 982-991. doi: 10.1111/desc. 12356

Pennington, B. F., and Lefly, D. L. (2001). Early reading development in children at family risk for dyslexia. Child Dev. 72, 816-833.

Peterson, R. L., and Pennington, B. F. (2015). Developmental dyslexia. Annu. Rev. Clin. Psychol. 11, 283-307. doi: 10.1146/annurev-clinpsy-032814-112842

Pugh, K., and Verhoeven, L. (2018). Introduction to this special issue: dyslexia across languages and writing systems. Sci. Stud. Read. 22, 1-6. doi: 10.1080/ 10888438.2017.1390668

R Core Team, (2018). R: A Language and Environment for Statistical Computing. Vienna: R Foundation for Statistical Computing.

Rosenberg, N. A., Huang, L., Jewett, E. M., Szpiech, Z. A., Jankovic, I., and Boehnke, M. (2010). Genome-wide association studies in diverse populations. Nat. Rev. Genet. 11, 356-366. doi: 10.1038/nrg2760

Shepley, C., and Grisham-Brown, J. (2019). Multi-tiered systems of support for preschool-aged children: a review and meta-analysis. Early Child. Res. Q. 47, 296-308. doi: 10.1016/j.ecresq.2019.01.004

Shu, H., McBride-Chang, C., Wu, S., and Liu, H. Y. (2006). Understanding Chinese developmental dyslexia: morphological awareness as a core cognitive construct. J. Educ. Psychol. 98, 122-133. doi: 10.1037/0022-0663.98.1.122
Skeide, M. A., Kirsten, H., Kraft, I., Schaadt, G., Muller, B., Neef, N., et al. (2015). Genetic dyslexia risk variant is related to neural connectivity patterns underlying phonological awareness in children. Neuroimage 118, 414-421. doi: 10.1016/j.neuroimage.2015.06.024

Skeide, M. A., Kraft, I., Muller, B., Schaadt, G., Neef, N. E., Brauer, J., et al. (2016). NRSN1 associated grey matter volume of the visual word form area reveals dyslexia before school. Brain 139(Pt 10), 2792-2803. doi: 10.1093/brain/ aww153

Snowling, M. J. (2012). Changing concepts of dyslexia: nature, treatment and comorbidity. J. Child Psychol. Psychiatry 53, e1-e3. doi: 10.1111/j.1469-7610. 2009.02197.x

Snowling, M. J. (2013). Early identification and interventions for dyslexia: a contemporary view. J. Res. Spec. Educ. Needs 13, 7-14. doi: 10.1111/j.1471-3802. 2012.01262.x

Snowling, M. J., Hayiou-Thomas, M. E., Nash, H. M., and Hulme, C. (2019). Dyslexia and developmental language disorder: comorbid disorders with distinct effects on reading comprehension. J. Child Psychol. Psychiatry. doi: 10.1111/jcpp.13140 [Epub ahead of print].

Snowling, M. J., and Hulme, C. (2012). Annual research review: the nature and classification of reading disorders-a commentary on proposals for DSM5. J. Child Psychol. Psychiatry 53, 593-607. doi: 10.1111/j.1469-7610.2011. 02495.x

Stein, J. (2018). What is developmental dyslexia? Brain Sci. 8:26. doi: 10.3390/ brainsci8020026

Tan, L. H., Liu, H. L., Perfetti, C. A., Spinks, J. A., Fox, P. T., and Gao, J. H. (2001). The neural system underlying Chinese logograph reading. Neuroimage 13, 836-846. doi: 10.1006/nimg.2001.0749

Tan, L. H., Spinks, J. A., Eden, G. F., Perfetti, C. A., and Siok, W. T. (2005). Reading depends on writing, in Chinese. Proc. Natl. Acad. Sci. U.S.A. 102, 8781-8785. doi: $10.1073 /$ pnas. 0503523102

Wang, Y., Lam, S. S.-Y., Mo, J., and McBride-Chang, C. (2014). "Pinyin knowledge as a potentially important marker of early literacy development and impairment in Mainland Chinese children," in Understanding Developmental Disorders of Auditory Processing, Language and Literacy across Languages: International Perspectives, eds K. K. H. Chung, K. C. P. Yuen, and D. M. McInerney, (Charlotte: Information Age Publishing).

Wenling, L., Anderson, R. C., Nagy, W., and Houcan, Z. (2002). "Facets of metalinguistic awareness that contribute to Chinese Literacy," in Chinese Children's Reading Acquisition: Theoretical and Pedagogical Issues, eds L. Wenling, J. S. Gaffney, and J. L. Packard, (Boston, MA: Springer), 87-106.

Yin, L., and McBride, C. (2018). Unspoken knowledge: kindergarteners are sensitive to patterns in Chinese pinyin before formally learning it. Lang. Cogn. Neurosci. 33, 65-76. doi: 10.1080/23273798.2017.1360502

Yin, W. G., and Weekes, B. S. (2003). Dyslexia in Chinese: clues from cognitive neuropsychology. Ann. Dyslexia 53, 255-279.

Zhao, H., Zhang, B., Chen, Y., Zhou, X., and Zuo, P. (2016). Environmental risk factors in han and uyghur children with dyslexia: a comparative study. PLoS One 11:e0159042. doi: 10.1371/journal.pone.0159042

Zhao, R., Fan, R., Liu, M., Wang, X., and Yang, J. (2017). Rethinking the function of brain regions for reading Chinese characters in a meta-analysis of fMRI studies. J. Neurolinguist. 44, 120-133. doi: 10.1016/j.jneuroling.2017. 04.001

Conflict of Interest: The authors declare that the research was conducted in the absence of any commercial or financial relationships that could be construed as a potential conflict of interest.

Copyright (c) $2020 \mathrm{Ma}$, Zhang, Hatfield and Wei. This is an open-access article distributed under the terms of the Creative Commons Attribution License (CC BY). The use, distribution or reproduction in other forums is permitted, provided the original author(s) and the copyright owner(s) are credited and that the original publication in this journal is cited, in accordance with accepted academic practice. No use, distribution or reproduction is permitted which does not comply with these terms. 